\title{
The immunomodulatory peptide bursopentin (BP5) enhances proliferation and induces sIgM expression in DT40 cells
}

\author{
Xiang-Bo Ji ${ }^{1,2, \#}$, Jun Luo, ${ }^{3, \#}$, Xiu-Li Feng ${ }^{1}$, Qiu-Liang $\mathrm{Xu}^{2}$, Teng Man ${ }^{3}$, Dong Zhao ${ }^{3}$, \\ Xin-Feng $\mathrm{Li}^{1}$, Gai-Ping Zhang ${ }^{3}$, Pu-Yan Chen ${ }^{1 *}$
}

1. Division of Key Lab of Animal Disease Diagnosis and Immunology of China's Department of Agriculture, Nanjing Agriculture University, Nanjing 210095, China.

2. Henan University of Animal Husbandry and Economy, Zhengzhou 450046, China.

3. Key Labaratory of Animal Immunology, Henan Academy of Agricultural Sciences, Zhengzhou 450002, China.

\# These authors contributed equally to this work.

\begin{abstract}
Background: In the recent past, many studies have been focused on extracts of BF and multiple biologically active factors and their effects on humoral immune system in chickens and birds. However, the mechanism of those immunomodulatory peptides on the $\mathrm{B}$ lineage cells proliferation and antibody production in chicken is fairly unknown. DT40 cell line, an avian leucosis virus-induced chicken pre-B cell line, expresses immunoglobulin $\mathrm{M}(\mathrm{IgM})$ isotype B cell reporter in the plasma membrane. There are many evidences suggesting that DT40 cells are best characterized as a bursal stem cell line. Because of the unique characteristics of DT40 cell line, it has been widely used to observe biological processes of pre-B lymphocyte cell within living cells.

Methods: The chicken B cell line DT40 was cultured in Roswell Park Memorial Institute (RPMI) 1640 medium and cytotoxicity was studied. Also, effect of BP5 on cell proliferation and cell cycle distribution of DT40 cells was studied. Also, the effect of BP5 on sIgM mRNA expression was studied by using real-time PCR.

Objectives: To investigat the effects of Bursopentin (Cys-Lys-Arg-Val-Tyr, BP5) on a chicken promyelocyte cell line DT40, assays of cell proliferation, cell cycle distribution, detection of surface immunoglobulin G (sIgM) mRNA expression and gene microarray analysis were performed.

Results: The results showed that BP5 displayed concentration-dependent effects on the proliferation, cell cycle, and sIgM mRNA expression in DT40 cells. And the analysis of expression profiles identified a signature set of 3022 genes (1254 up regulated genes, 1762 down regulated genes), which clearly discriminated the BP5-treated DT40 cells from control with high certainty $(\mathrm{P} \leq 0.02)$. The results of microarray analysis were confirmed by quantitative reverse transcription-polymerase chain reaction for 12 of the differentially expressed genes.

Conclusion: Theses findings showed the immuno-activity effect of BP5 on B lymphocyte and indicated that BP5 treatment regulated eight signaling pathways, in which Toll-like signaling pathway was the most significant enrichment pathway.
\end{abstract}

Keywords: Bursopentin (BP5), DT40 cell, Proliferation, Cell cycle, sIgM, gene microarray.

DOI: https://dx.doi.org/10.4314/ahs.v18i4.50

Cite as: Ji X-B, Luo J, Feng X-L, Xu Q-L, Man T, Zhao D, Li X-F, Zhang G-P, Chen P-Y. The immunomodulatory peptide bursopentin (BP5) enhances proliferation and induces sIgM expression in DT40 cells. Afri Health Sci. 2018;18(4): 1292-1302. https:/ / dx. doi.org/10.4314/ abs.v18i4.50

\section{Corresponding author:}

$\mathrm{Pu}-$ Yan Chen,

Division of Key Lab of Animal Disease

Diagnosis and Immunology of China's

Department of Agriculture,

Nanjing Agriculture University,

No.1 Weigang Street, Nanjing 210095, China,

Email: puyanchen4@gmail.com

\section{Introduction}

The bursa of fabricius (BF), or "cloacal thymus", is the primary lymphoid organ in birds. It plays a central role in the proliferation and differentiation of the antibody-producing B lymphocyte lineage ${ }^{1}$. Previous studies have shown that some defined peptides sequenced from a BF extract (e.g., bursin and bursal anti-steroidogenic peptide) stimulate specific immune cell subsets ${ }^{2,3}$ isolated a new pentapeptide, bursopentin (BP5; Cys-Lys-Arg-Val-Tyr), 
from a BF extract. BP5 was shown to have immunomodulatory effects, including effects on T and B cells, the antioxidant stress response of macrophages, and an inhibitory effect on tumor cell proliferation.

Many studies have focused on BF extracts and multiple biologically active factors and their effects on the humoral immune system in chickens and other birds ${ }^{2}$. However, the mechanisms through which these immunomodulatory peptides affect B lineage cell proliferation and antibody production in chickens is poorly understood. DT40 cells are an avian leukosis virus-induced chicken pre-B cell line that express the immunoglobulin $\mathrm{M}$ ( $\mathrm{IgM}$ ) isotype B cell reporter in the plasma membrane. DT40 cells are best characterized as a bursal stem cell line ${ }^{4}$. Given the unique characteristics of DT40 cells, they have been widely used to observe the biology of pre-B lymphocytes within living cells.

In the present study, the effects of BP5 on the proliferation and cell cycle of DT40 cells were investigated. Furthermore, the role of BP5 in the expression of surface IgM ( $\operatorname{sgM}$ ) mRNA was determined by real-time polymerase chain reaction (PCR). A gene microarray analysis of DT40 cells treated with or without BP5 was performed to further understand the potential effect of BP5 on pre-B cell development. Signaling pathway and Gene Ontology (GO) analyses were also performed to identify potential signaling pathways involved in these BP5-mediated effects.

\section{Materials and methods \\ Cell lines and culture}

BP5 was synthesized by the Key Laboratory of Animal Immunology of the Ministry of Agriculture (Henan, China). The purity of the synthetic peptide was $>99 \%$; this was confirmed by reverse-phase high-performance liquid chromatography. DT40 cells were cultured in Roswell Park Memorial Institute (RPMI) 1640 medium (Gibco, Grand Island, NY,USA) containing 10\% fetal bovine serum (FBS; Gibco) and 5\% chicken serum (CHS; Gibco) supplemented with $50 \mu \mathrm{M} \beta$-mercaptoethanol, penicillin $(100 \mathrm{IU} / \mathrm{ml})$, and streptomycin $(100 \mu \mathrm{g} / \mathrm{ml})$. The cells were incubated in a humidified incubator containing 5\% $\mathrm{CO}_{2}$ at $37^{\circ} \mathrm{C}$.

\section{Effects of BP5 on DT40 cell proliferation}

DT40 cells were allowed to grow until reaching 0.4-0.6 $\times 10^{6}$ cells $/ \mathrm{ml}$. Then, the cells were collected and washed three times with RPMI 1640 basic medium. The cells were then incubated in tissue culture flasks at different densities $\left(1,2\right.$, and $3 \times 10^{6}$ cells $\left./ \mathrm{ml}\right)$ with varying concentrations $(0.02,0.2,2$, and $20 \mu \mathrm{g} / \mathrm{ml})$ of BP5 in RPMI 1640 medium (final volume, $10 \mathrm{ml}$ ) containing 1\% FBS plus $1 \%$ CHS or $10 \%$ FBS plus $5 \%$ CHS, respectively, for $96 \mathrm{~h}$. The cells were harvested at $0,24,48$, and $72 \mathrm{~h}$ post-seeding and quantified using a hemocytometer under a light microscope (Olympus, Tokyo, Japan). Cell viability was assessed by the trypan blue exclusion assay. All experiments were performed in triplicate.

\section{Effects of BP5 on the cell cycle distribution of DT40 cells}

DT40 cells $\left(2 \times 10^{6}\right.$ cells $\left./ \mathrm{ml}\right)$ were incubated in tissue culture flasks with varying concentrations $(0.02,0.2,2$, and $20 \mu \mathrm{g} / \mathrm{ml}$ ) of BP5 in RPMI 1640 medium (final volume, $10 \mathrm{ml}$ ) containing 1\% FBS plus 1\% CHS for $72 \mathrm{~h}$. The cells were collected 24,48 , and $72 \mathrm{~h}$ post-seeding, washed twice with cold phosphate-buffered saline (PBS), resuspended in $70 \%$ cold ethanol, and incubated at $4{ }^{\circ} \mathrm{C}$ for $2 \mathrm{~h}$. Then, the cells were washed with cold PBS and incubated in $0.5 \mathrm{ml}$ of propidium iodide (PI)-binding buffer $(50 \mu \mathrm{g} /$ $\mathrm{ml}$ of PI and $100 \mu \mathrm{g}$ of ribonuclease) at $37^{\circ} \mathrm{C}$ for $30 \mathrm{~min}$. The cell cycle distribution of DT40 cells was determined on a FACSCaliber flow cytometer with CellQuest Pro analysis software (Becton-Dickinson, Brea, CA, USA). The experiments were performed in triplicate.

\section{Effects of BP5 on sIgM mRNA expression as shown by real-time PCR}

Cells were treated with varying concentrations of BP5 $(0.02,0.2,2$, and $20 \mu \mathrm{g} / \mathrm{ml})$ and collected 24,48 , and 72 $\mathrm{h}$ post-seeding. Total RNA was isolated using TRIzol reagent (Invitrogen, Carlsbad, CA, USA) according to the manufacturers' instructions. To obtain complementary DNA (cDNA), $2 \mu \mathrm{g}$ of total RNA was reverse transcribed using a reverse transcription kit (Takara Bio, Shiga, Japan) according to the manufacturer's protocol. Each PCR mixture contained $1 \mu$ l of template cDNA, $10 \mu$ of $2 \times$ SYBR ${ }^{\circledR}$ Green PCR Master Mix, and 5 pmol each of the forward and reverse primers (final volume, $25 \mu \mathrm{l}$ ). The 
sIgM and control $\beta$-actin transcripts were amplified using real-time PCR as per a standard protocol $\left(15 \mathrm{~s}\right.$ at $95^{\circ} \mathrm{C}$ and $30 \mathrm{~s}$ at $60^{\circ} \mathrm{C}$ for 40 cycles, respectively). All reactions were run in triplicate. Relative expression of the sIgM gene was determined according to the $2-\Delta \Delta$ CT formula. Mean efficiency values were calculated from the amplification profiles of individual samples using LinRegPCR software.

\section{Gene microarray and data analyses}

DT40 cells were incubated for $4 \mathrm{~h}$ in the presence or absence of BP5. Total RNA was obtained as described above. The samples were labelled and hybridized according to the Agilent One-Color Microarray-Based Gene Expression Analysis protocol (Agilent Technologies, Palo Alto, CA, USA). The microarray data were deposited in a Gene Expression Omnibus database under Accession Number GSE30396. The resulting text files were extracted using Agilent Feature Extraction software (ver. 10.5.1.1) and imported into Agilent GeneSpring GX software (ver. 11.0) for further analysis. To determine the genes that were most robustly regulated by BP5, a foldchange threshold of $\geq 2$ was applied and compared with control cells treated with PBS.

\section{Statistical analysis}

All data are presented as the mean \pm standard deviation. A P-value $<0.05$ was considered significant. Student's t-test was used for the analysis.

\section{Results}

\section{BP5 has dual effects on DT40 cell proliferation}

The effect of BP5 on DT40 cell proliferation in two different media varied. In the growth medium $(10 \% \mathrm{FBS}$ plus $5 \%$ CHS), the DT40 cells exhibited a rapid increase in number, and BP5 showed no significant effect on the growth curves of DT40 cells (data not shown). However, in the maintenance medium (1\% FBS plus 1\% CHS), BP5 treatment resulted in dual effects: proliferation (0.02 and $0.2 \mu \mathrm{g} / \mathrm{ml}$ ) and the inhibition of proliferation (2 and 20 $\mu \mathrm{g} / \mathrm{ml}$ ) of DT40 cells 24 and 48 h post-seeding (Fig. 1).
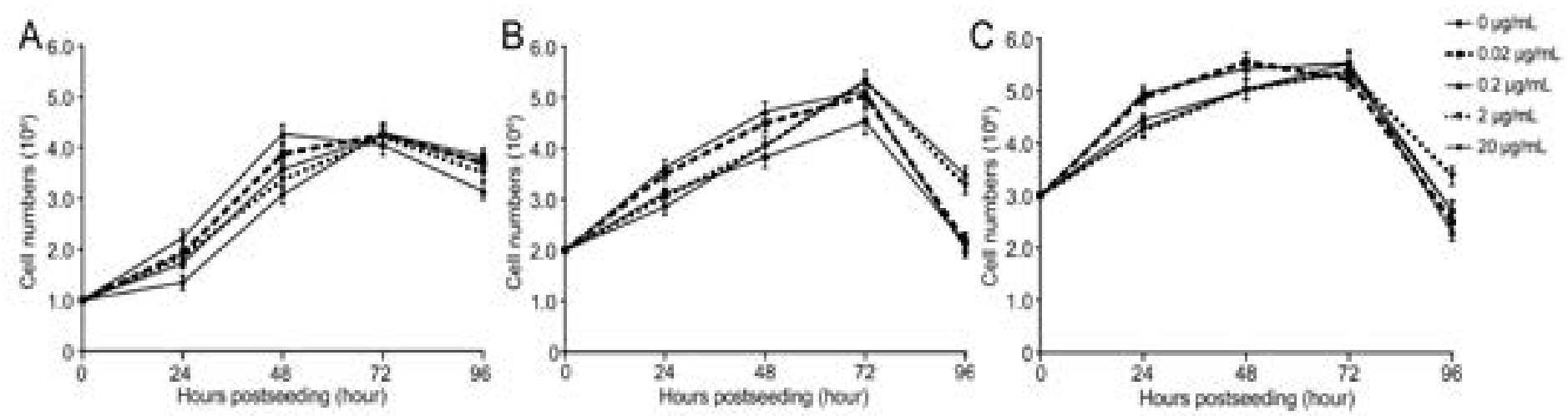

Fig.1 


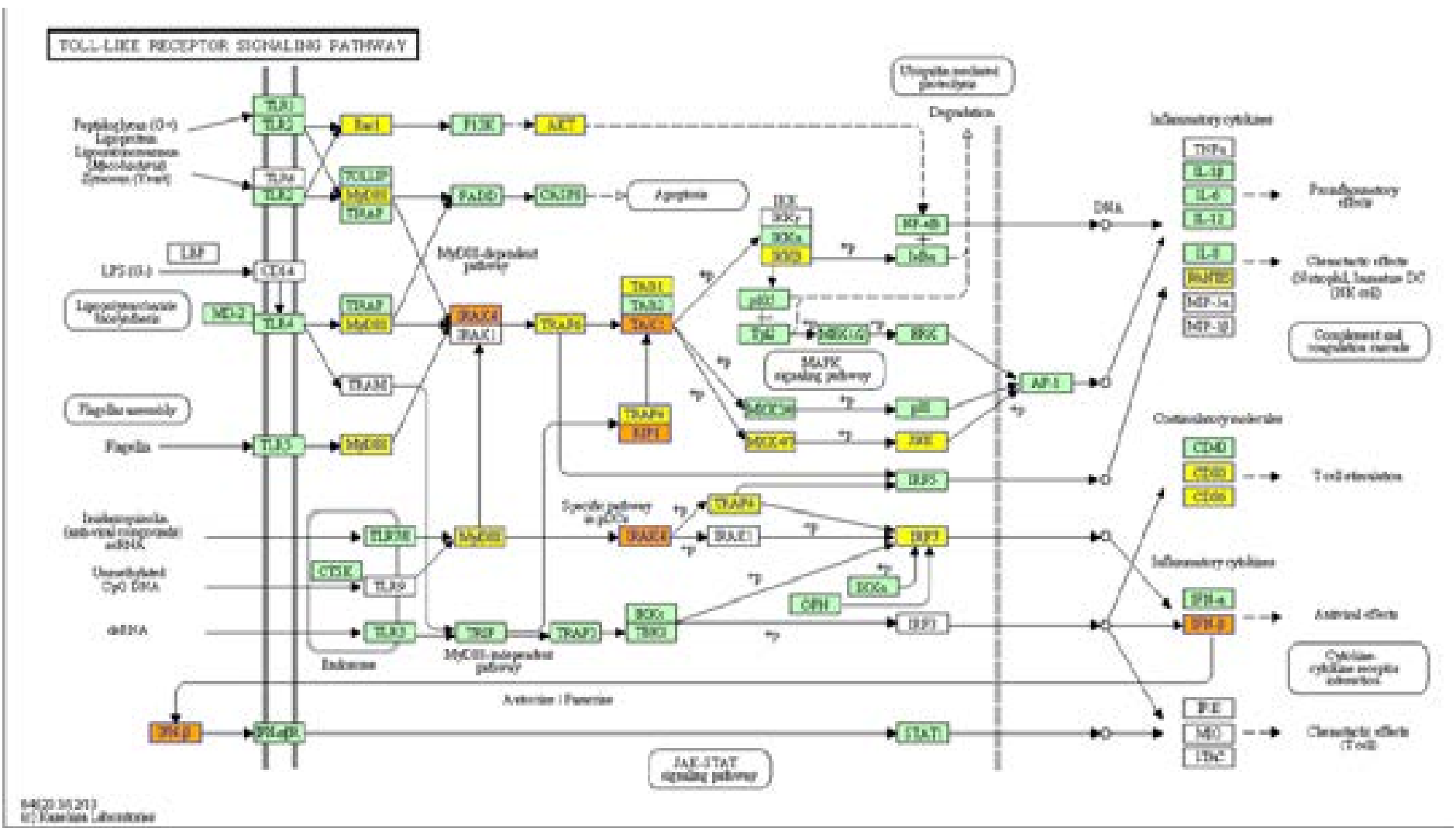

Fig. S1

The cell counts in the 0.02 and $0.2 \mu \mathrm{g} / \mathrm{ml} \mathrm{BP5-treated}$ cells were lower than that in the control group, whereas the cell number following treatment with $20 \mu \mathrm{g} / \mathrm{ml}$ of BP5 was higher than that in the control group $72 \mathrm{~h}$ post-seeding. These data indicate that BP5 displays concentration-dependent dual effects on the proliferation of DT40 cells.

\section{Effects of BP5 on the DT40 cell cycle distribution}

As shown in Fig. 2, the percentage of cells in $\mathrm{S}$ phase decreased in all groups when the incubation period was extended. Moreover, the percentages of cells in S phase in the 0.02 and $0.2 \mu \mathrm{g} / \mathrm{ml} \mathrm{BP5}$ treatment groups were significantly higher than that in the control group at 24 and $48 \mathrm{~h}$, respectively. The percentages of cells in $\mathrm{G} 2 / \mathrm{M}$ phase in the 2 and $20 \mu \mathrm{g} / \mathrm{ml} \mathrm{BP5}$ treatment groups were higher than that in the control group. These results indicate that 0.02 or $0.2 \mu \mathrm{g} / \mathrm{ml}$ of BP5 treatment promoted cell cycle accumulation in the $\mathrm{S}$ phase, whereas 2 or 20 $\mu \mathrm{g} / \mathrm{ml}$ of BP5 treatment arrested the cell cycle in G2/M phase, consistent with the results of the cell number and viability assays.

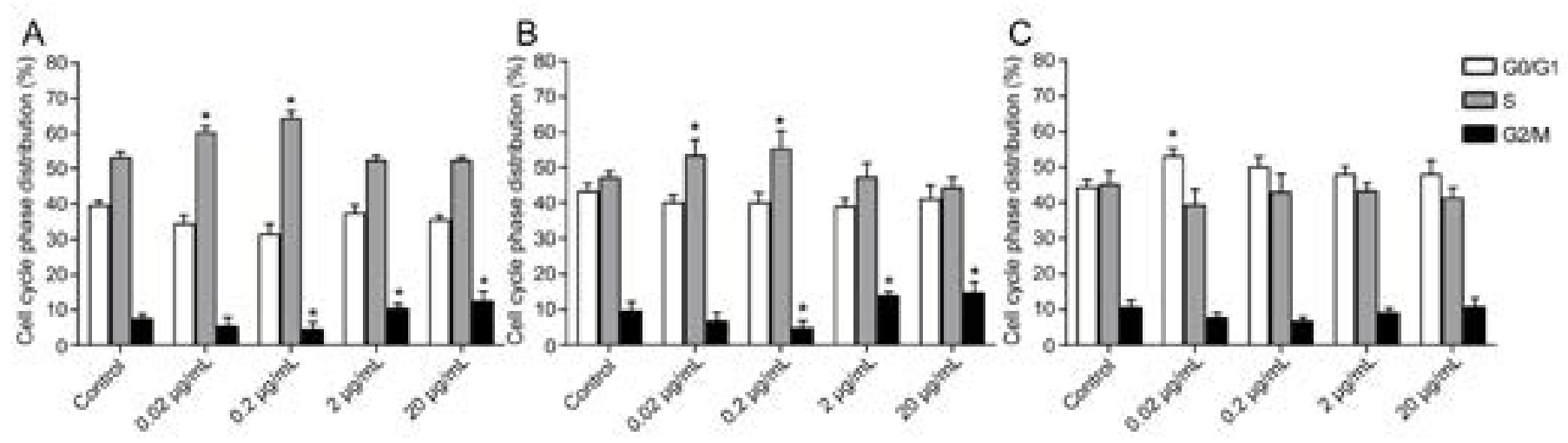

Fig.2 


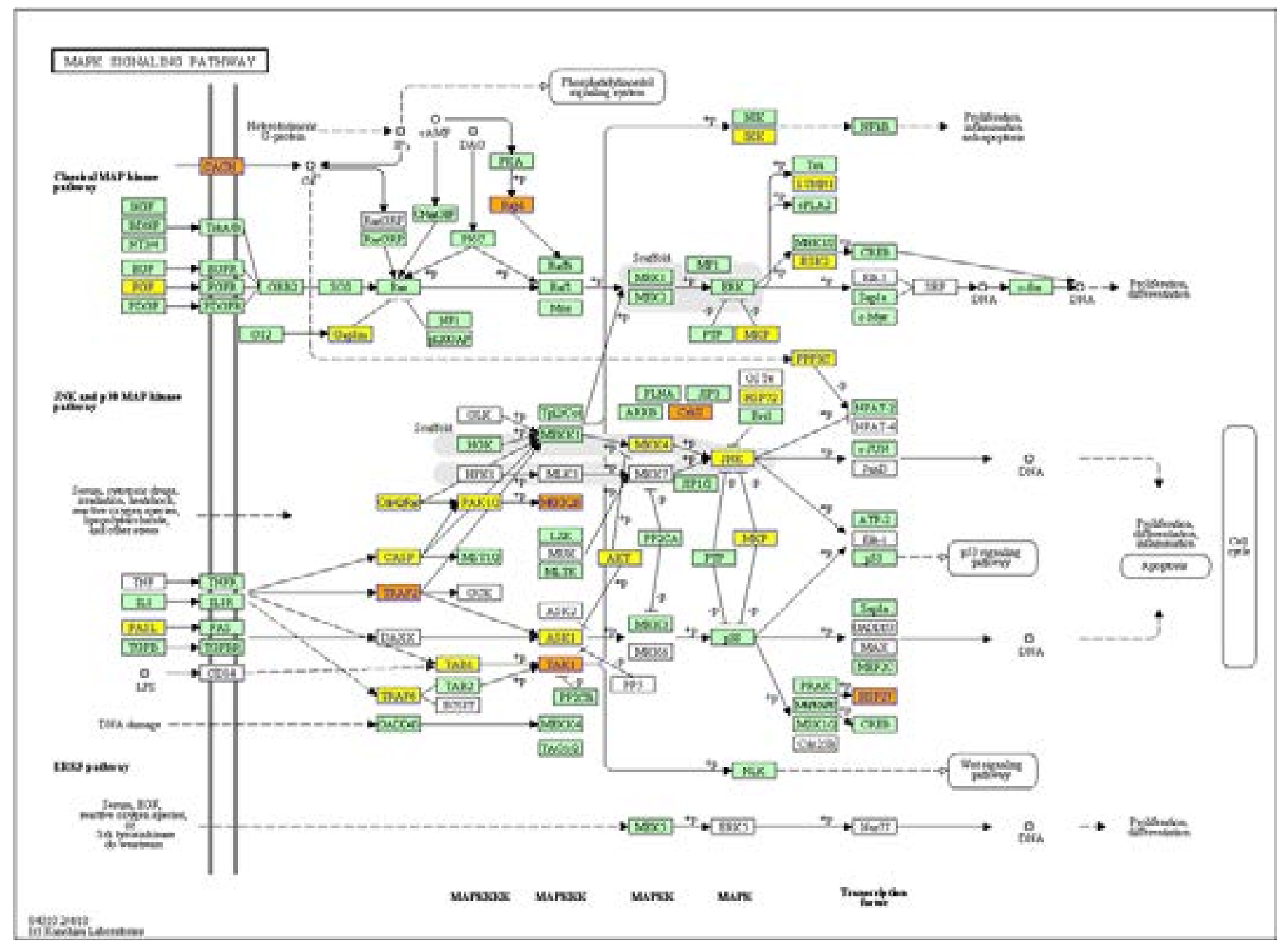

Fig. S2

Effects of BP5 on sIgM mRNA expression in DT40 cells

The relative sIgM mRNA levels increased in all BP5 treatment groups (Fig. 3). In particular, the sIgM mRNA lev$\mathrm{el}$ at the $20 \mu \mathrm{g} / \mathrm{ml}$ concentration was significantly higher

than that in the control group. The increase in sIgM gene expression was enhanced concentration-dependently at $24 \mathrm{~h}$, whereas it declined at $48 \mathrm{~h}$, with no further difference in sIgM expression among these groups at $72 \mathrm{~h}$.

Analysis of gene expression profiling in BP5-treated DT40 cells

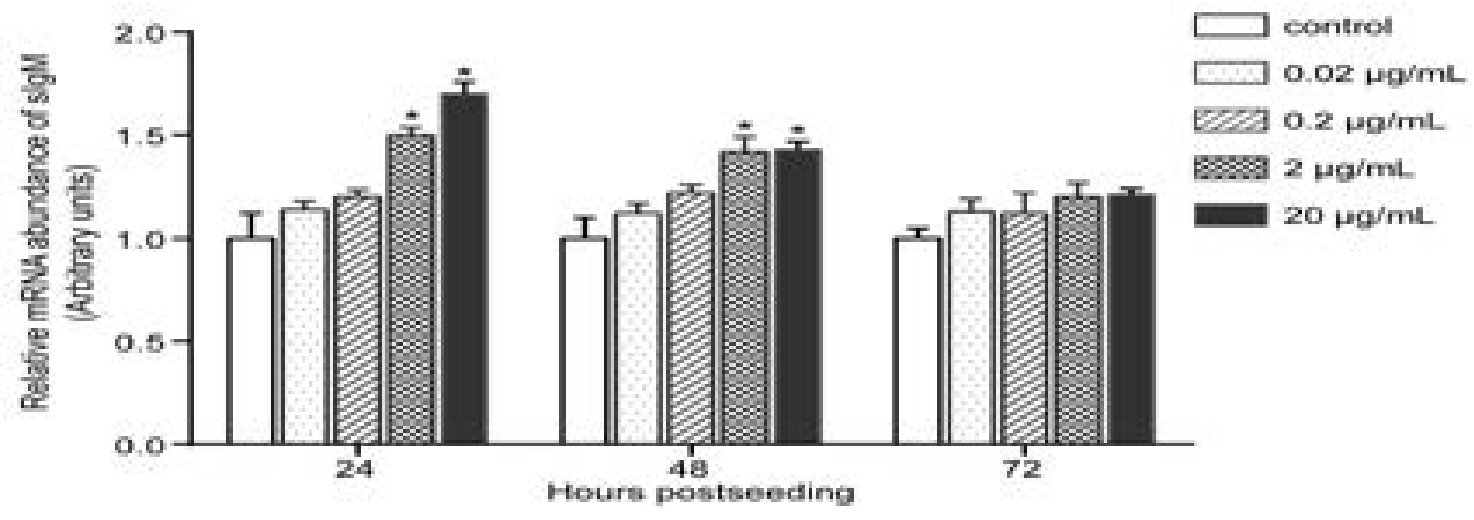

Fig.3 


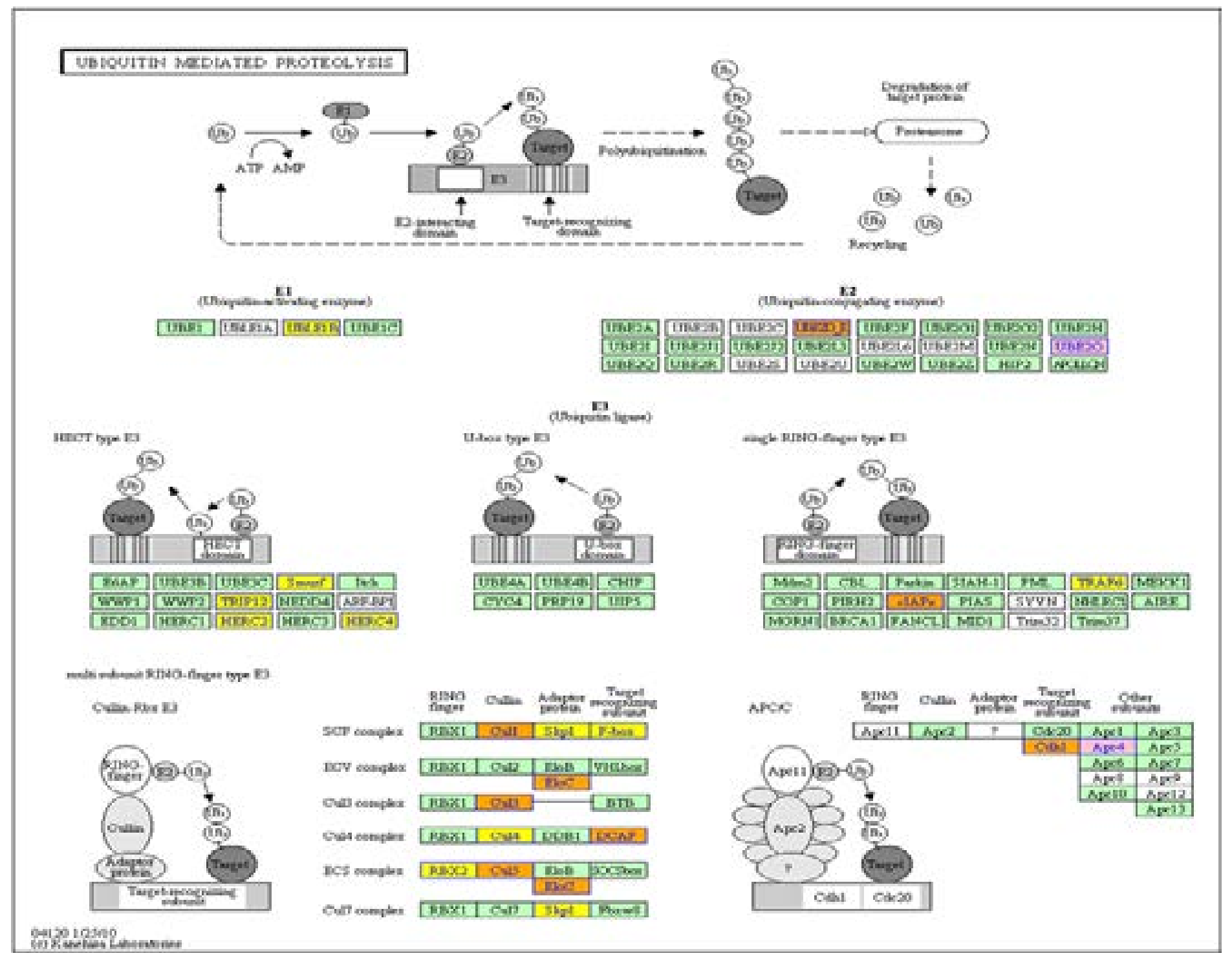

Fig. S3

A gene expression profile of DT40 cells after $4 \mathrm{~h}$ of incubation with $0.02 \mu \mathrm{g} / \mathrm{ml}$ of BP5 was carried out to further identify the molecular mechanism underlying the effects of BP5 on pre-B lymphocyte biology. As shown in the scatterplot (Fig. 4A), genes above the top green line and below the bottom green line showed a 2.0-fold change, and variations in expression were detected between the treated and control cells. Gene expression profiling revealed that 1,254 genes were upregulated and 1,762 genes were downregulated after BP5 treatment. We also used real-time reverse transcription-PCR (qRT-PCR) to validate the microarray results. The primer sequences of the twelve selected genes are shown in Table S1. 


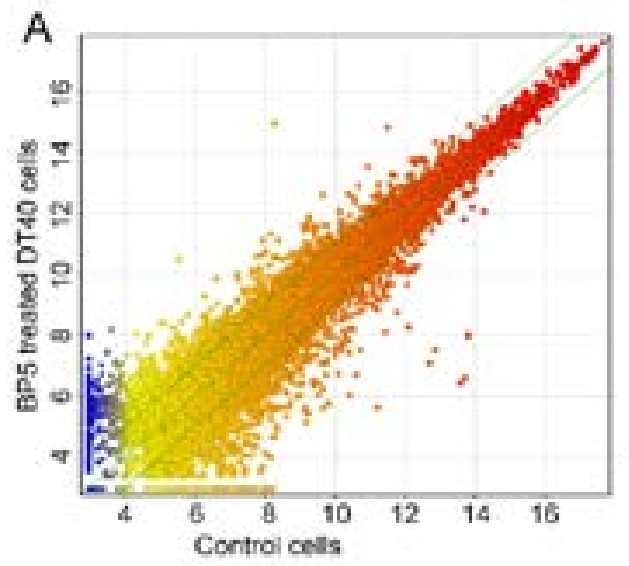

B

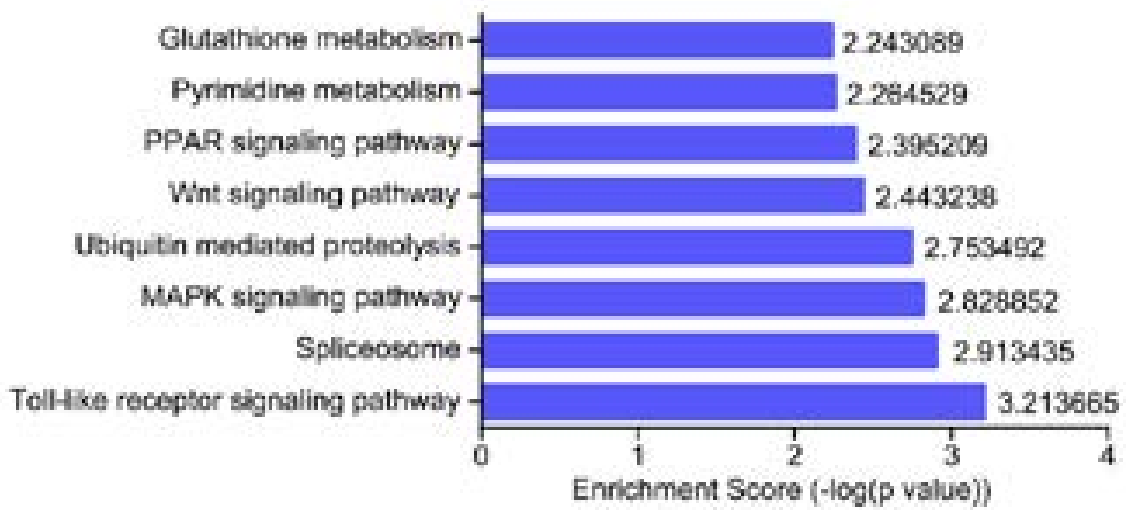

Fig.4

WNT SIGLLANG PATHWhY

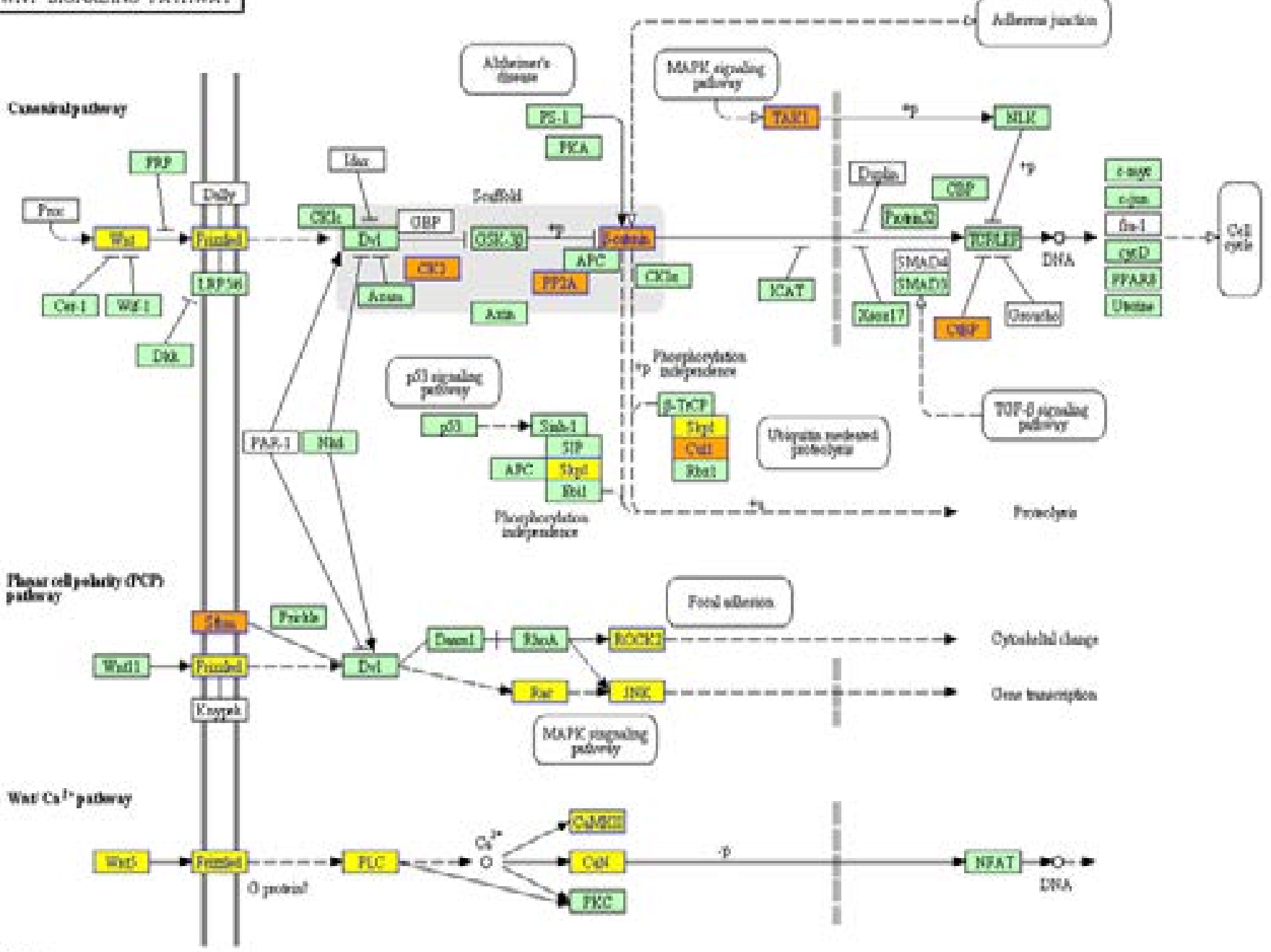

\section{Fig.S4}


Table S1. The primer sequences of the selected genes

\begin{tabular}{|c|c|}
\hline Genes & Primer sequences ( $5^{\prime}$ to $\left.3^{\prime}\right)$ \\
\hline \multirow{2}{*}{ GAPDH } & TTGACCTGACCTGCCGTCTG \\
\hline & TGGCTGTCACCATTGAAGTCAC \\
\hline \multirow{2}{*}{ FZR1 } & TCCTGGGAGCAGGGATTGAG \\
\hline & GACTGGAACGTTTGGTGCTGAG \\
\hline \multirow{2}{*}{ HDAC1 } & AGGACTGCCCTGTGTTCGATG \\
\hline & TCCTGCCCAATTCACAGCAA \\
\hline \multirow{2}{*}{ GDF3 } & AGAGACCCGGTGGATGGTTGTA \\
\hline & ATGGTCAGGCGCTCATCCTC \\
\hline \multirow{2}{*}{ FGF3 } & TGTCGGGATCGTCGCTATCA \\
\hline & GGTACAGACGGGATGCATAGGTG \\
\hline \multirow{2}{*}{ IRF7 } & TGAAGGTCAACACACCACAGGAG \\
\hline & CCCAACCACAAAGCTTATTGCAG \\
\hline \multirow{2}{*}{ DNMT1 } & AGTCGTGGTTCCAGAGGCAGA \\
\hline & CACCAAAGCGCTCATGTCCTTA \\
\hline \multirow{2}{*}{ CSNK2A1 } & CTCCTGTCAGCAGTGCGAGTATG \\
\hline & ATGACGGGTGAGCCTGCTAGA \\
\hline \multirow{2}{*}{ SKP1 } & TTAAGCTGCAGAGTTCAGATGGAGA \\
\hline & CTGGGTCATCATCGCCTTCA \\
\hline \multirow{2}{*}{ RAP1B } & CAAGCTAGTGGTTCTTGGTTCTGGA \\
\hline & GCATACACTGTTGCGCATCTACTTC \\
\hline \multirow{2}{*}{ TCEB1 } & AGAGCATGCATTAACATCAGGAACA \\
\hline & GGTGCAATTGGGAATTCAGGA \\
\hline \multirow{2}{*}{ FGF8 } & TCATCGTCGAGACCGACACC \\
\hline & CAGTCCTTGCCTTTGCCGTTA \\
\hline \multirow{2}{*}{ TRIP12 } & GTTTATCATGAGCCCATTCCAACAG \\
\hline & TTCCAGCCACATATTAGCAGTGACA \\
\hline
\end{tabular}

Signaling pathway analysis of differentially expressed genes

Signaling pathway and GO analyses were performed to identify the biological functions of the subsets of differentially expressed genes after BP5 treatment. Enriched genes associated with signaling pathways were identified according to the Kyoto Encyclopedia of Genes and Genomes database. Eight signaling pathways were affected by BP5 treatment, including the Toll-like receptor (TLR) signaling pathway, mitogen-activated protein kinase (MAPK) signaling pathway, ubiquitin-mediated proteolysis, and Wnt signaling pathway (Table 1 and Fig. 4B, more information is shown in Figs. S1-4). Most of these signaling pathways are involved in pre-B lymphocyte development and humoral immunity.

African Health Sciences Vol 18 Issue 4, December, 2018

\section{TLR signaling pathway}

Toll-induced B cell proliferation may be an ancient pathway of the innate immune system ${ }^{5}$. The TLR signaling pathway was the most significant pathway with the highest enrichment score (3.214) in DT40 cells after BP5 treatment. Some important genes, including those encoding interferon-beta (IFN- $\beta$ ), IRAK4, and mitogen-activated protein kinase 7 (MAP3K7), were upregulated, whereas IRF7, MYD88, and Ras-related C3 botulinum toxin substrate 1 (RAC1) were downregulated, after PB5 treatment. These results suggest that BP5, which has been reported to be an immunomodulatory factor in birds, produces diverse innate immune responses.

MAPK signaling pathway

The MAPK pathway mediates multiple cellular responses, 
including cell survival, proliferation, differentiation, and apoptosis ${ }^{6}$. Except for mitogen-activated protein kinase 3 and RAC1, the expression of fibroblast growth factor (FGF) 3 and RAP1B were induced by BP5 treatment, whereas the expression of FGF8, mitogen-activated protein kinase 4, mitogen-activated protein kinase 5, and P21 protein activated kinase 2 (PAK2) was downregulated. The enrichment score for this pathway was 2.829, suggesting that BP5 regulates B cell development through the MAPK signaling pathway.

\section{Ubiquitin-mediated proteolysis}

Ubiquitin-mediated proteolysis plays an important role in basic cellular processes such as regulating the cell cycle, modulating immune and inflammatory responses, and controlling signal transduction pathways ${ }^{7}$. In this study, the expression of several genes, including those encoding FZR1, transcription elongation factor B (SIII), and transcription elongation factor B (SIII), polypeptide 1 (TCEB1), were upregulated, whereas the expression of SKP1, thyroid hormone receptor interactor 12, F-Box protein 2, and HERC4 were downregulated after BP5 treatment.

\section{Wnt signaling pathway}

The Wnt signaling pathway plays an important role in cell development and oncogenesis ${ }^{8}$. The expression of genes such as MAP3K7, CSNK2A1, and CTNNB1 was upregulated, whereas the expression of RAC1, SKP1, and calcium binding protein p22 was downregulated after PB5 treatment.

\section{Discussion}

$\mathrm{BF}$ is a unique humoral immune organ of birds that plays an important role in B cell differentiation and antibody production. Increasing attention has been focused on identifying new biopeptides from the BF, and there is increasing evidence that these peptides play a vital role in the B cell array of basic cellular processes ${ }^{9}$. A previous study demonstrated that bursal-derived BP5 is a multifunctional immune-inducing factor that upregulates antibody production ${ }^{3}$. The present results confirmed the effects of BP5 on cell proliferation, the cell cycle, and sIgM mRNA expression in the avian pre-B cell line DT40. Interestingly, BP5 displayed concentration-dependent dual effects on DT40 cells. A low concentration of BP5 induced DT40 proliferation, while a high concentration of
BP5 inhibited DT40 proliferation. The results of the cell cycle and apoptosis investigations were comparable with the DT40 cell growth curve. The low concentration of BP5 induced S phase at $24 \mathrm{~h}$, while the high concentration of BP5 arrested the cell cycle in $\mathrm{G} 2 / \mathrm{M}$ phase. A high concentration of BP5 could result in increased apoptosis of DT40 cells (data not shown). It has been reported that cross-linking sIgM on B cells results in different signaling consequences: relatively weak cross-linking of sIgM induces DNA synthesis, whereas intense cross-linking of sIgM results in apoptotic cell death ${ }^{3}$. In the present study, sIgM mRNA expression was concentration-dependently enhanced after BP5 treatment. However, the association between enhanced sIgM mRNA expression and DT40 cell apoptosis should be confirmed in future studies.

The gene expression profile of DT40 cells after $4 \mathrm{~h}$ of incubation with $0.02 \mu \mathrm{g} / \mathrm{ml}$ of BP5 was determined via gene microarray analysis to further identify the molecular mechanism of BP5 in pre-B lymphocyte biological processes. Cellular signaling pathway analyses indicated that eight signaling pathways were affected by BP5 treatment, including pathways associated with the cell cycle, cell proliferation, and cytokine secretion. Considering that BP5 has dominant effects on immune responses, the present study focused on four signaling pathways associated with immunity and cell development.

TLR-mediated signaling is broadly classified into MyD88-dependent and -independent pathways. TLR-mediated intracellular signal transduction involves utilization of the signaling molecule MyD88, followed by the nuclear translocation of $\mathrm{NF} x \mathrm{~B}$, activation of the protein kinase B/Akt, extracellular signal-regulated kinase, c-Jun N-terminal kinase, and MAPK, and phosphorylation of the IRF transcription factor family ${ }^{10}$. IRAKs are important mediators of TLR-induced intracellular signal transduction as they potentiate downstream signaling. Some studies indicate that IRAK4 and IRAK1 are sequentially phosphorylated and dissociated from MyD88 upon stimulation, resulting in the activation of tumor necrosis factor receptor-associated factor $6^{11}$. In the present study, the MyD88 gene was downregulated in DT40 cells treated with BP5. The IRF7 gene, which was recently identified as a key regulator of type I interferon (IFN) activation, was also downregulated. The IRAK4 and IFN- $\beta$ genes were upregulated via MyD88-independent pathways, which are associated with the JNK-STAT signaling path- 
way. These findings indicate that BP5 may regulate IFN- $\beta$ secretion via MyD88-independent pathways rather than through IRF7.

Given the importance of cross-talk between signaling pathways, it was not surprising that multiple genes (e.g., FGF3, FGF8, and RAP1B) associated with the MAPK and Wnt pathways were affected in BP5-treated DT40 cells. FGF3 and FGF8 are key regulators of biological responses during cell development, and MAPK signaling is the most widely known downstream pathway used by FGF3 and FGF8 during cell developmental processes ${ }^{12}$. In the present study, FGF3 and RAP1B were upregulated, while FGF8 was downregulated, in BP5-treated DT40 cells. Furthermore, PAK2, a key factor in the JNK and p38 MAP kinase pathways that is highly associated with cell apoptosis, was downregulated. Moreover, CTNNB1 and CSNK2A1 were induced in BP5-treated DT40 cells, whereas RAC1 was downregulated. $\beta$-Catenin, a subunit of the cadherin protein complex, is an intracellular signal transducer that regulates coordination of cell-cell adhesion and gene transcription ${ }^{13}$. CSNK2A1 is a subunit of casein kinase 2, which is involved in suppressing apoptosis and cell survival. CSNK2A1 induces resting $\mathrm{B}$ cells to enter the cell cycle ${ }^{14}$. Protein kinase CK2 alpha is overexpressed in colorectal cancer and modulates cell proliferation and invasion by regulating EMT-related genes $^{15}$. These results indicate that BP5 may affect DT40 cell development, including cell proliferation, apoptosis, and antibody production by triggering a complex signaling network.

Additionally, the ubiquitin-mediated proteolysis pathway was significantly enriched, and genes involved in this pathway, including FZR1 and TCEB1, were upregulated, whereas SKP1 and F-BOX2 were repressed in BP5-treated DT40 cells. FZR1 is an important regulator of G1 phase and the loss of it would shorten G1 and lengthen S phase ${ }^{16}$. SKP1 is a core component of the SKP Cullin F-box protein complex, a type of ubiquitin ligase that initiates the cell division cycle via the timely destruction of many regulatory proteins. In the present study, the percentage of cells in S phase increased following treatment with $0.02 \mu \mathrm{g} / \mathrm{ml}$ of BP5 compared to control cells, in agreement with previous results. These results indicate that BP5 may regulate genes that control the DT40 cell cycle, resulting in B cell development.

\section{Conclusion}

In summary, the present results demonstrate that BP5 exerts a strong regulatory effect on DT40 cell biological processes. The results of the gene microarray analysis revealed the gene expression profile and various signaling pathways mediating the effects of bursal-derived peptides in B cell development. In future, the identified key regulatory genes should be studied to verify the signaling pathways associated with BP5-induced effects.

\section{Acknowledgements}

We are grateful to the Key Laboratory of Animal Immunology of Henan Academy of Agricultural Sciences for providing BP5 and the DT40 cells. This study was supported by a grant from the National Natural Science Foundation of China (No. 31302067).

\section{Conflicts of interest}

The authors have no conflicts of interest.

\section{References}

1. Audhya T, Kroon D, Heavner G, Viamontes G, Goldstein G. Tripeptide structure of bursin, a selective B-cell-differentiating hormone of the bursa of fabricius. Science. 1986; 231: 997-999. PubMed.

2. Feng XL, Liu QT, Cao RB, Zhou B, Ma ZY, Deng WL, Wei JC, Qiu YF, Wang FQ, Gu JY, Wang FJ, Zheng QS, Ishag H, Chen PY. Identification and characterization of novel immunomodulatory bursal-derived pentapeptide-II (BPP-II). J Biol Chem. 2012; 287: 3798-3807

3. Li DY, Geng ZR, Zhu HF, Wang C, Miao DN, Chen PY. Immunomodulatory activities of a new pentapeptide (Bursopentin) from the chicken bursa of Fabricius. Amino Acids. 2011; 40: 505-515. PubMed.

4. Gerondakis S, Grumont RJ, Banerjee A. Regulating B-cell activation and survival in response to TLR signals. Immunol Cell Biol. 2007; 85: 471-475. PubMed.

5. Bonet C, Giuliano S, Ohanna M, Bille K, Allegra M, Lacour J-P, Bahadoran P, Rocchi S, Ballotti R, Bertolotto C. Aurora $\mathrm{B}$ is regulated by the mitogen-activated protein kinase/extracellular signal-regulated kinase (MAPK/ ERK) signaling pathway and is a valuable potential target in melanoma cells. Journal of Biological Chemistry. 2012; 287: 29887-29898

6. Zhang Z, Miao L, Lv C, Sun H, Wei S, Wang B, Huang C, Jiao B. Wentilactone B induces G2/M phase arrest and apoptosis via the Ras/Raf/MAPK signaling pathway in 
human hepatoma SMMC-7721 cells. Cell death \& disease. 2013; 4: 657

7. Peng X, Yang L, Chang H, Dai G, Wang F, Duan X, Guo L, Zhang Y, Chen G. Wnt/ $\beta$-catenin signaling regulates the proliferation and differentiation of mesenchymal progenitor cells through the p53 pathway. 2014; 9(5): 97283

8. Feng X, Liu T, Wang F, Cao R, Zhou B, Zhang Y, Mao X, Chen P, Zhang H. Isolation, anti-proliferation on tumor cell and immunomodulatory activity of BSP-I, a novel bursal peptide from chicken humoral immune system. Peptides. 2011; 32: 1103-1109. PubMed.

9. Feng X, Cao R, Zhou B, Liu Q, Liu K, Liu X, Zhang Y, Gu J, Miao D, Chen P. The potential mechanism of Bursal-derived BPP-II on the antibody production and avian pre-B cell. Vaccine. 2013; 31: 1535-1539. PubMed.

10. Kawai T, Akira S. TLR signaling. Cell Death \& Differentiation. 2006; 13: 816-825. PubMed.

11. Arsura M, Hofmann CS, Golay J, Introna M, Sonenshein GE. A-myb rescues murine B-cell lymphomas from IgM-receptor-mediated apoptosis through c-myc transcriptional regulation. Blood. 2000; 96: 1013-1020. PubMed.

12. Dailey L, Ambrosetti D, Mansukhani A, Basilico C. Mechanisms underlying differential responses to FGF signaling. Cytokine \& growth factor reviews. 2005; 16: 233-247 13. Brembeck FH, Rosário M, Birchmeier W. Balancing cell adhesion and Wnt signaling, the key role of $\beta$-catenin. Current opinion in genetics \& development. 2006; 16: 51-59. PubMed.

14. Debenedette M, Snow EC. Induction and regulation of casein kinase II during B lymphocyte activation. The Journal of Immunology. 1991; 147: 2839-2845

15. Zou J, Luo H, Zeng Q, Dong Z, Wu D, Liu L. Protein kinase $\mathrm{CK} 2 \mathrm{a}$ is overexpressed in colorectal cancer and modulates cell proliferation and invasion via regulating EMT-related genes. J Transl Med. 2011; 9: 97

16. DeSalle LM, Pagano M. Regulation of the G1 to S transition by the ubiquitin pathway. FEBS letters. 2001; 490: 179-189. PubMed. 